\title{
Somatosensory evoked potential monitoring detection of carotid compression during ACDF surgery in a patient with a vascularly isolated hemisphere
}

\author{
Alan D. Legatt, MD, PhD, ${ }^{1,2}$ Avra S. Laarakker, MD, ${ }^{3}$ Jonathan P. Nakhla, MD, ${ }^{4}$ Rani Nasser, MD, ${ }^{4}$ \\ and David J. Altschul, MD4
}

Departments of ${ }^{1}$ Neurology, ${ }^{2}$ Neuroscience, and ${ }^{4}$ Neurosurgery, Montefiore Medical Center and the Albert Einstein College of Medicine, Bronx, New York; and ${ }^{3}$ Ruth \& Bruce Rappaport Faculty of Medicine, Technion-Israel Institute of Technology, Haifa, Israel

The authors report herein a case of anterior cervical discectomy and fusion (ACDF) surgery in which findings on somatosensory evoked potential (SSEP) monitoring led to the correction of carotid artery compression in a patient with a vascularly isolated hemisphere (no significant collateral blood vessels to the carotid artery territory). The amplitude of the cortical SSEP component to left ulnar nerve stimulation progressively decreased in multiple runs, but there were no changes in the cervicomedullary SSEP component to the same stimulus. When the lateral (right-sided) retractor was removed, the cortical SSEP component returned to baseline. The retraction was then intermittently relaxed during the rest of the operation, and the patient suffered no neurological morbidity. Magnetic resonance angiography demonstrated a vascularly isolated right hemisphere.

During anterior cervical spine surgery, carotid artery compression by the retractor can cause hemispheric ischemia and infarction in patients with inadequate collateral circulation. The primary purpose of SSEP monitoring during ACDF surgery is to detect compromise of the dorsal column somatosensory pathways within the cervical spinal cord, but intraoperative SSEP monitoring can also detect hemispheric ischemia. Concurrent recording of cervicomedullary SSEPS can help differentiate cortical SSEP changes due to hemispheric ischemia from those due to compromise of the dorsal column pathways. If there are adverse changes in the cortical SSEPs but no changes in the cervicomedullary SSEPS, the possibility of hemispheric ischemia due to carotid artery compression by the retractor should be considered.

http://thejns.org/doi/abs/10.3171/2016.4.SPINE151481

KEY WORDS anterior cervical discectomy and fusion; carotid artery compression; circle of Willis; intraoperative neurophysiological monitoring; isolated hemisphere; somatosensory evoked potentials; technique

I NTRAOPERATIVE monitoring (IOM) of somatosensory evoked potentials (SSEPs) and motor evoked potentials (MEPs) is used to assess the integrity of the spinal cord during cervical decompression and fusion surgery. Following stimulation of the median nerve or the ulnar nerve at the wrist, both cortically and subcortically generated SSEP components can be recorded. These components are named for their polarity and typical latency in healthy adults; for example, P14 is a positivity with a typical peak latency of $14 \mathrm{msec}$. The P14 component is generated at the level of the cervicomedullary junction (cuneate nucleus), and the
N20 component is generated in the primary somatosensory cortex. ${ }^{7}$ Within the spinal cord, these SSEPs are mediated by the dorsal columns, not the spinothalamic tracts, ${ }^{2}$ and spinal cord compromise would typically affect both cortical and subcortical SSEPs. We report herein a case of anterior cervical discectomy and fusion (ACDF) surgery during which the cortical and subcortical SSEPs to left ulnar nerve stimulation were differentially affected. This IOM finding led to the correction of carotid artery compression, and angiography subsequently demonstrated that the patient had a vascularly isolated right cerebral hemisphere.

ABBREVIATIONS ACDF = anterior cervical discectomy and fusion; $\mathrm{ACOA}=$ anterior communicating artery; $\mathrm{BP}=\mathrm{blood}$ pressure; $\mathrm{CCA}=\mathrm{common}$ carotid artery; $\mathrm{EMG}=$ electromyographic; ICA = internal carotid artery; IOM = intraoperative monitoring; $M E P=$ motor evoked potential; MRA = MR angiogram; PCA = posterior cerebral artery; $\mathrm{PCOA}=$ posterior communicating artery; SSEP = somatosensory evoked potential; TES = transcranial electrical stimulation.

SUBMITTED December 15, 2015. ACCEPTED April 19, 2016.

INCLUDE WHEN CITING Published online June 10, 2016; DOI: 10.3171/2016.4.SPINE151481. 


\section{Case Report}

History

A 51-year-old man saw a neurologist for a chief complaint of severe left retromastoid headaches that had been occurring for the past 5 years with increasing frequency, as well as radiating pain and numbness in his left arm. He also reported left greater than right arm weakness while lifting weights. The patient had no history of atherosclerosis, stroke, or neurovascular procedures. He was not being treated with aspirin.

\section{Examination}

Magnetic resonance imaging showed a herniated C6-7 disc, asymmetrical to the left, with severe spinal cord compression; there was also a mild disc bulge at the C5-6 level without spinal cord compression. The patient was referred for neurosurgical treatment. Anterior cervical discectomy and fusion surgery was performed at the C6-7 level with intraoperative neurophysiological monitoring. All ACDF surgeries at our hospital are done with neurophysiological monitoring.

On examination, hand intrinsic muscle strength was Medical Research Council grades $4+/ 5$ on the left and $5 / 5$ on the right. Strength was $5 / 5$ bilaterally in the other tested upper-limb muscles: deltoid, biceps, triceps, wrist extensors, finger extensors, and finger flexors. Biceps and triceps reflexes were asymmetrical, $2+$ on the right and $1+$ on the left. Hoffman's sign was absent bilaterally, but a positive Spurling's sign was present on the left. On sensory examination, light touch was decreased in the C-7 dermatome on the left. Strength in the legs was $5 / 5$ bilaterally. Knee jerks were symmetrical but were brisk bilaterally. Gait and station were normal.

\section{Monitoring Techniques}

Cortical and cervicomedullary SSEPs to unilateral stimulation of each ulnar nerve at the wrist and of each posterior tibial nerve at the ankle were recorded from needle electrodes in the scalp; the same electrodes were used for continuous electroencephalographic monitoring. Motor evoked potentials to transcranial electrical stimulation (TES) of the brain were recorded from needle electrodes in the biceps-triceps, thenar-hypothenar, and abductor hallucis muscles bilaterally; the TES parameters were titrated to produce large and clear MEPs in all monitored muscle groups contralateral to the TES anode for both stimulus polarities. Spontaneous electromyographic (EMG) activity was monitored bilaterally from needle electrodes in the trapezius-deltoid, biceps-triceps, and thenar-hypothenar muscles and from laryngeal electrodes on the surface of the endotracheal tube.

\section{Surgery and IOM Findings}

After localizing the C6-7 level with fluoroscopy, a right-sided transverse incision was made ventral to the anterior border of the sternocleidomastoid muscle, and blunt dissection was performed, exposing the anterior spine. The longus colli muscles were undermined with monopolar electrocautery on either side, and self-retaining retractors were then inserted. The dissection was done medial to the sternocleidomastoid muscle to avoid both entering the carotid sheath and requiring lateral retraction of the carotid artery to visualize the disc. The endotracheal tube was deflated for placement of the retractors and reinflated after the retractors were in place. The retractors (TrimLine self-retaining retractors, Medtronic Sofamor Danek) were stable in their positions; they did not migrate.

At baseline, clear cortical and cervicomedullary SSEPs were present following stimulation of either ulnar nerve (Fig. 1). As the surgery progressed, there was progressive attenuation of the cortical SSEPs to left ulnar nerve stimulation that approached 50\% of the baseline amplitude (the usual alarm level for SSEP changes), without a corresponding attenuation of the cervicomedullary SSEPs to the same stimulus (Figs. 2 and 3). The surgeons and the monitoring team discussed the SSEP changes and possible etiologies for them. Since the pattern of SSEP changes was more consistent with brain dysfunction than with spinal cord dysfunction, we considered the possibility that right hemispheric perfusion was being compromised due to compression of the right common carotid artery (CCA). The retractor was removed; this was followed by a substantial increase in the cortical SSEP (waveform at 17:24 in Fig. 2). The retractor was replaced, and the cortical SSEP to left ulnar nerve stimulation decreased in amplitude again. Because of these findings, the retractor was intermittently removed several times during the remainder of the operation so that carotid compression would not be prolonged. The total time that the retractors were in place was approximately 40 minutes. The amplitude of the cortical SSEP to left ulnar nerve stimulation never went below $50 \%$ of its baseline amplitude. The amplitude of the cortical SSEP to right ulnar nerve stimulation did show some downward trend during the early part of the operation, but it never went below $75 \%$ of its baseline amplitude. No significant adverse changes in the cortical SSEPs to posterior tibial nerve stimulation were noted during the operation, but they were recorded less often and were not sampled during the time of the most prominent changes in the ulnar nerve SSEPs. There were no clear changes in the MEPs, aside from a transient increase in the amplitude of the MEP in the left biceps-triceps recording channel at 17:25, approximately the same time as the SSEP increase (Fig. 4). This change was well within the range of normal MEP run-to-run variability, however.

The patient was not hypotensive as the ulnar nerve SSEPs were progressively attenuating; mean arterial blood pressure (BP), recorded every 5 minutes, ranged from 81 to $92 \mathrm{~mm} \mathrm{Hg}$. Thus, no pressors were given. A single mean $\mathrm{BP}$ of $62 \mathrm{~mm} \mathrm{Hg}$ was recorded at 17:20, but it went up to 95 $\mathrm{mm} \mathrm{Hg}$ in the next measurement.

The discectomy was performed, including removal of the posterior spur-disc complex down to the posterior longitudinal ligament. A zero-profile anchored spacer was then inserted into the disc space and attached to the vertebral bodies above and below using screws under fluoroscopic guidance. The total duration of the operation was approximately 2 hours.

\section{Postoperative Course}

The patient had no new neurological deficits following 
Left Ulnar Nerve SEPs

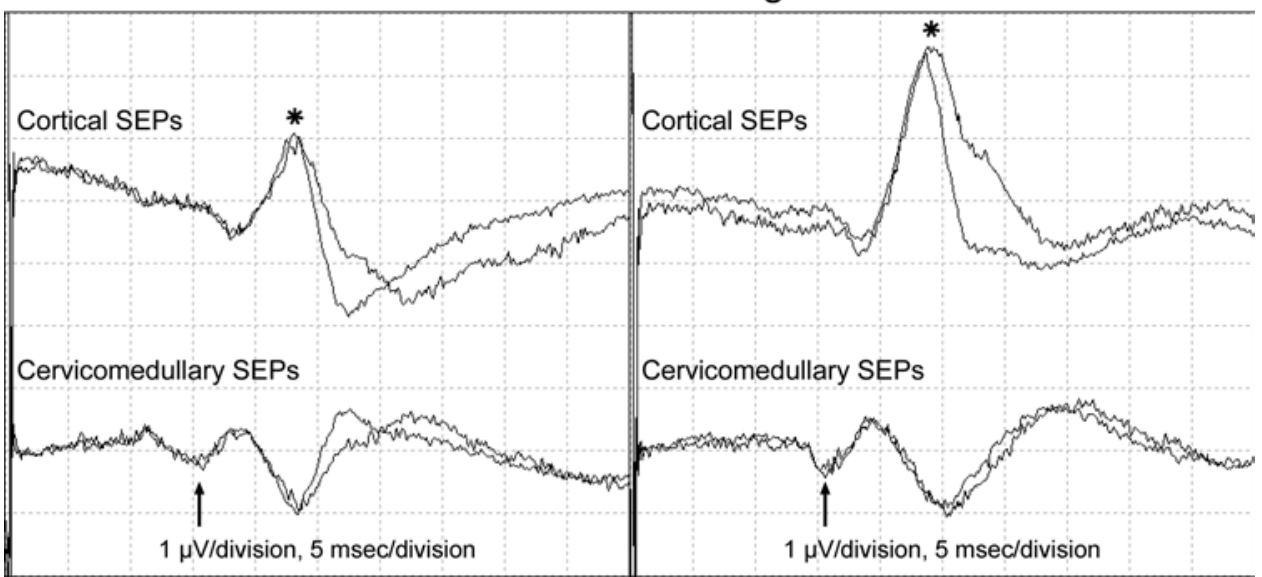

FIG. 1. Somatosensory evoked potentials (SEPS) to ulnar nerve stimulation, recorded at the beginning of the operation, with 2 runs superimposed. Asterisks indicate N20, the primary cortical SSEP component. Arrows indicate P14, the cervicomedullary SSEP component; the cervicomedullary recording channels also pick up a second peak, which represents inverted volume-conducted cortical SSEPs. Negativity is shown by an upward deflection.

the surgery and fared well. Prior to discharge, an MR angiogram (MRA; Fig. 5) demonstrated the absence of the anterior communicating artery $(\mathrm{ACoA})$ and a fetal right posterior cerebral artery (PCA) originating from the right internal carotid artery (ICA). The left PCA originated from the basilar artery, and a left posterior communicat- ing artery (PCoA) was present. The MRA did not show significant atherosclerotic disease of the carotid arteries.

\section{Discussion}

During ACDF surgery in a patient without significant

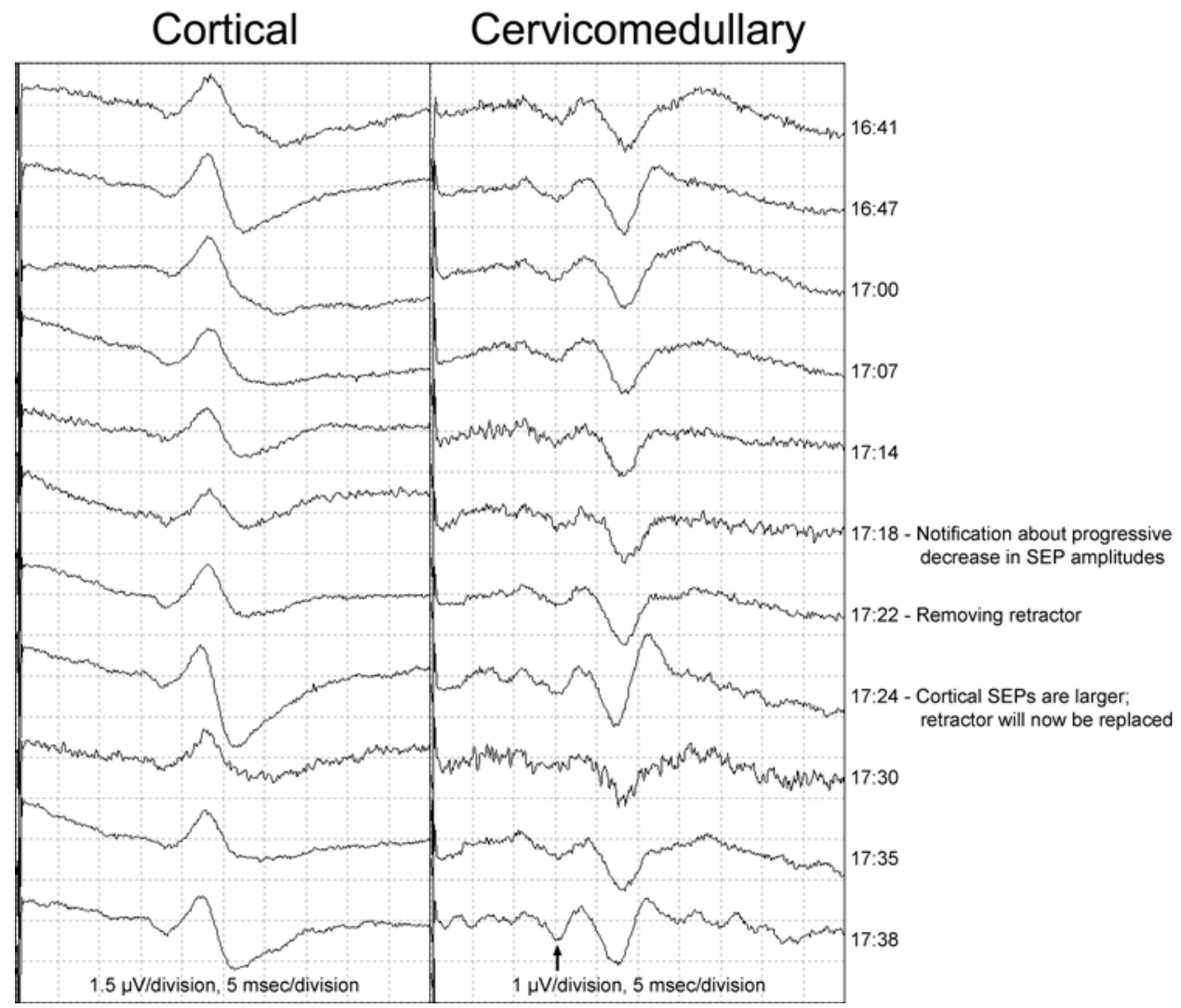

FIG. 2. Cortical (N20) and cervicomedullary (P14) SSEPs to left ulnar nerve stimulation showing the intraoperative changes. Arrow indicates P14, the cervicomedullary SSEP component; longer-latency peaks representing inverted volume-conducted cortical SSEPs are also visible in this recording channel. Negativity is shown by an upward deflection. 


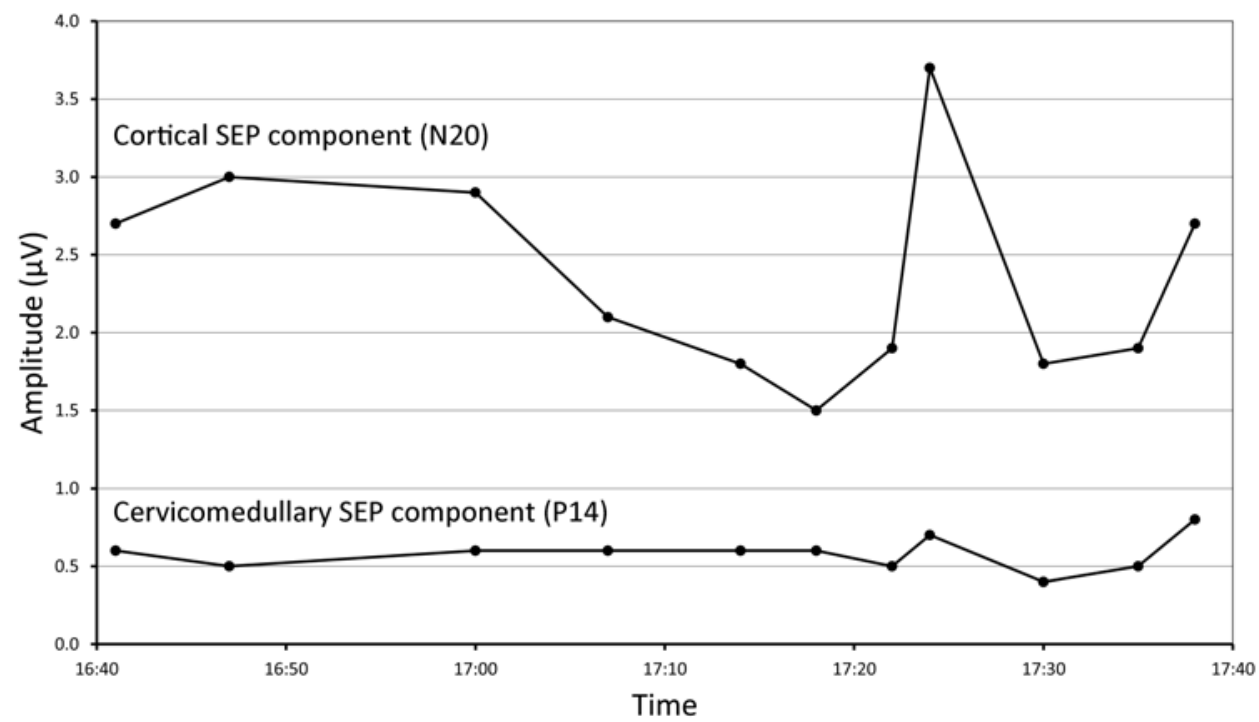

FIG. 3. Graph of the amplitude measurements of the N20 and P14 components of the SSEPs to left ulnar nerve stimulation, from the waveforms in Fig. 2. The amplitude measurements were taken from the peak of the component to the following peak of the opposite polarity. Note that the P14 amplitudes were stable during the progressive attenuation of the N20 amplitudes.

atherosclerosis, SSEPs showed changes that were consistent with hemispheric ischemia rather than with spinal cord dysfunction, raising the possibility of carotid artery compression. Removing the retractors led to recovery of the SSEPs. The retractors were then intermittently removed several times during the remainder of the operation so that there would not be prolonged carotid compression, and the patient suffered no neurological morbidity.
A postoperative MRA demonstrated a vascularly isolated right hemisphere, explaining why compression of the right carotid artery had caused right hemispheric ischemia.

The carotid artery compression in this case was due to compression by the retractors, but the compression was not caused by migration of the retractors. During ACDF surgery, some carotid artery compression by the lateral retractor is inevitable. Using Doppler ultrasound measure-

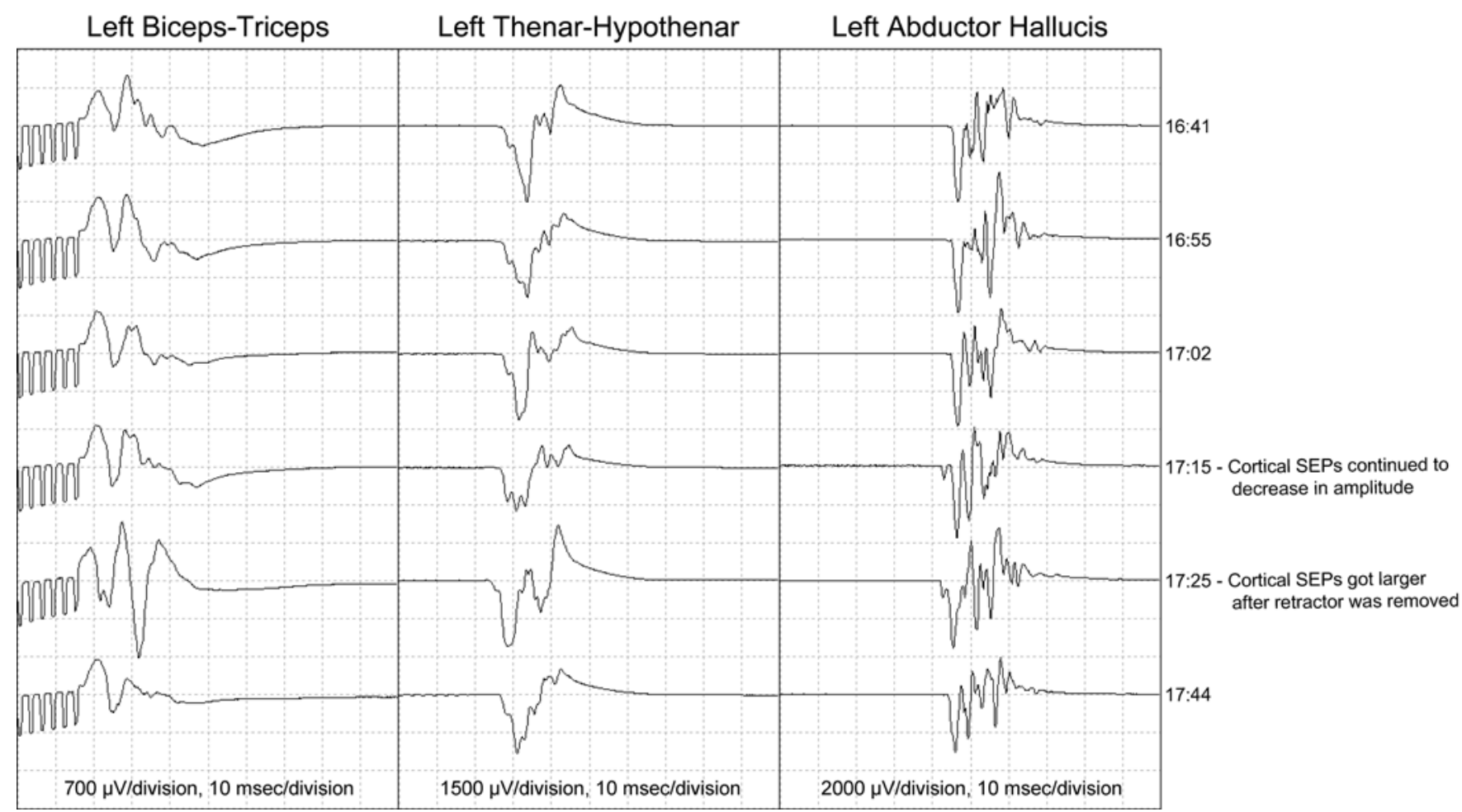

FIG. 4. Intraoperative MEPs to anodal stimulation of the right hemisphere. The transient increase in the amplitude of the left biceps-triceps MEPs at 17:25 is well within the range of typical MEP run-to-run variability. 


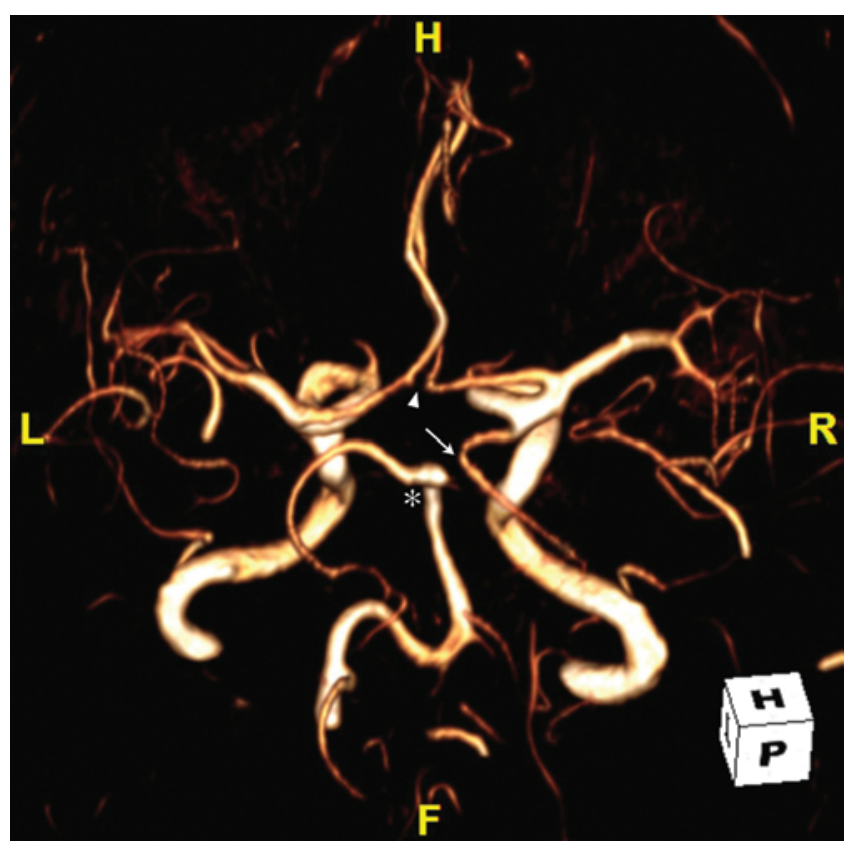

FIG. 5. Three-dimensional reconstruction of the intracranial vasculature, derived from the postoperative time-of-flight MRA. Note the absence of the ACoA (arrowhead). The left PCA arises from the top of the basilar artery (asterisk). However, the right PCA (arrow) arises from the right carotid artery, and there is no communication between it and the basilar artery. Figure is available in color online only.

ments of carotid blood velocities during a series of 15 operations, Pollard and Little ${ }^{8}$ found that the average reduction in the cross-sectional area of the carotid artery was $30 \%$. Individual patient results were not included in that paper, so the intersubject variability of the carotid compression and its maximum value were not reported. They did note that the average cross-sectional area reduction was higher (55\%) in the subset of 3 patients younger than 40 years old, and they theorized that the lack of atherosclerosis made these carotid arteries less rigid and more compressible. In contrast, there was almost no carotid artery compression in the oldest patients, that is, those over 70 years of age. ${ }^{8}$ The lack of significant carotid artery atherosclerotic disease in our patient may have made his right carotid artery more susceptible to compression.

Can carotid artery compression by the lateral retractor cause an ischemic cerebral injury? There are rare reports of strokes ipsilateral to the retractor during anterior cervical spine surgery. Drummond et al. $^{3}$ reported one such case in which the effects of carotid compression may have been exacerbated by intraoperative hypotension; an MRA study in their patient demonstrated the bilateral absence of the PCoAs and a hypoplastic ACoA, similar to the vascular pattern in our patient. Yeh at al. ${ }^{12}$ reported a massive, fatal ipsilateral hemispheric stroke following surgery for revision of an ACDF; there was no intraoperative hypotension, but the surgery was prolonged (5 hours) and the retraction had been readjusted but never relaxed completely during it; no information about collateral vessels was provided. Chozick et al. ${ }^{1}$ reported a delayed ipsilateral stroke occurring on the 3rd postoperative day. Angiography showed a complete ipsilateral carotid thrombosis, which the authors speculated might have been initiated by stasis within a compressed carotid artery. No information about neurophysiological monitoring data were included in these 3 case reports.

Sloan et al. ${ }^{11}$ reported the transient unilateral disappearance of cortical SSEPs generated in the hemisphere ipsilateral to the retractor in 3 patients having anterior cervical spine surgery, one of whom had had radical neck surgery with contralateral carotid ligation and radiation therapy as part of her treatment for squamous cell carcinoma. The anatomy of the cerebral vasculature was not described in the other 2 patients; in one of them, identifiable cervicomedullary SSEPs were present and were unchanged when the cortical SSEPs deteriorated. In all 3 patients, repositioning of the retractors reversed the cortical SSEP changes. Our patient is the first reported case of SSEP monitoring demonstrating reversible cerebral ischemia from carotid compression by the retractor with documentation of the predisposing cerebrovascular anatomy.

Presumably, the lower-limb SSEPs would have also shown adverse changes in this patient, but they were not sampled during the time of the most prominent changes in the ulnar nerve SSEPs. The lack of adverse MEP changes (Fig. 4) may have reflected the titration of the TES parameters to produce large and clear MEPs in all monitored muscle groups. This may stimulate the corticospinal tract axons as far caudally as the brainstem:10 the MEPs elicited by such stimulation would not be affected by hemispheric ischemia.

The primary purpose of SSEP monitoring during cervical spine surgery is to detect dysfunction of the dorsal column pathways within the cervical spinal cord. For that purpose, recording either the N20 components, generated in the primary somatosensory cortex following ulnar nerve stimulation, or the P14 components, generated at the level of the dorsal column nuclei, would suffice. Recording both components provides a measure of redundancy, in case one is, or becomes, unusable due to EMG noise accompanying a light depth of anesthesia (which would predominantly affect the cervicomedullary SSEPs), attenuation due to a deep level of anesthesia (which would predominantly affect the cortical SSEPs), ${ }^{6}$ or technical problems. As demonstrated in our patient, recording both components also permits one to distinguish different causes of deterioration of the cortical SSEPs.

Dorsal column dysfunction would be expected to affect both the P14 and N20 components. In our patient, the stability of the P14 component to left-sided stimulation indicated that there was no dorsal column dysfunction and that the concurrent N20 attenuation reflected dysfunction of the intracranial somatosensory pathways rostral to the level of the dorsal column nuclei. Since this could reflect cerebral ischemia due to compression of the right CCA, the retractor was removed; this resulted in a substantial increase in the cortical SSEP amplitude, with a subsequent decrease when the retractor was replaced. Because of this finding, the retractor was intermittently removed several times during the remainder of the operation to avoid prolonged hemispheric ischemia due to carotid compression. The patient had no new postoperative neurological deficits.

A postoperative MRA demonstrated that our patient 
had a vascularly isolated right hemisphere due to the absence of the ACOA as well as a right fetal PCA (Fig. 5). A complete circle of Willis in which no component is hypoplastic or absent is seen in only $20 \%-25 \%$ of individuals. Common normal variants include hypoplasia of one or both $\mathrm{P}_{1}$ segments, a hypoplastic or absent $\mathrm{A}_{1}$ segment of the anterior cerebral artery, and a fetal origin of the PCA. Reported rates of unilateral fetal PCA range from $4 \%$ to $29 \%$; a fetal PCA occurs bilaterally in $1 \%-9 \%$ of cases. ${ }^{4}$ Definitive absence of the ACoA is the least common variation in the circle of Willis; it has been found in $5 \%$ of surgical dissections. ${ }^{?}$

The prevalence of the definitive absence (or functional absence due to severe hypoplasia) of the ACoA in combination with a fetal PCA resulting in isolated hemispheric circulation has not been reported but can be assumed to be very low given the low prevalence of each finding independently. Our patient has a totally isolated right hemisphere precisely due to this combination. Given the rarity of this pattern of vascular anomalies, we believe that a routine preoperative MRA is not warranted in patients who will undergo ACDF surgery, especially if the surgery will be performed with SSEP monitoring, which can detect hemispheric ischemia.

Although the attenuation of cortical SSEPs in our patient did not quite reach the typical alarm criterion of $50 \%$, notification was given because there was progressive amplitude attenuation. When IOM data are showing progressively worsening changes, it may be appropriate to raise an alert before they reach a specific predefined threshold level. ${ }^{5}$ The carotid compression in our patient may not have been complete, allowing the hemisphere to tolerate the hypoperfusion for several minutes. The intermittent release of retractors kept the hypoperfusion from reaching a point that could cause neurological damage. While it was not done during this case, elevation of the systemic blood pressure could also help to increase hemispheric perfusion in other patients in whom SSEP monitoring detects hemispheric ischemia during ACDF surgery, especially if systemic hypotension appears to be a contributing factor. As a prophylactic measure, wide platysma and longus colli dissection can help to prevent added pressure of the carotid artery from the retraction.

\section{Conclusions}

During anterior cervical spine surgery, carotid artery compression by the retractor can cause hemispheric ischemia and infarction in patients with inadequate collateral circulation. Intraoperative SSEP monitoring can detect hemispheric ischemia, leading to adjustments in surgical procedures to prevent infarction. Recording cervicomedullary SSEPs can help differentiate cortical SSEP changes due to hemispheric ischemia from those due to compromise of the dorsal column pathways within the spinal cord. If there are adverse changes in the cortical SSEPs but no changes in the cervicomedullary SSEPs, the possibility of hemispheric ischemia due to carotid artery compression by the retractor should be considered.

\section{References}

1. Chozick BS, Watson P, Greenblatt SH: Internal carotid artery thrombosis after cervical corpectomy. Spine (Phila Pa 1976) 19:2230-2232, 1994

2. Cusick JF, Myklebust JB, Larson SJ, Sances A Jr: Spinal cord evaluation by cortical evoked responses. Arch Neurol 36:140-143, 1979

3. Drummond JC, Englander RN, Gallo CJ: Cerebral ischemia as an apparent complication of anterior cervical discectomy in a patient with an incomplete circle of Willis. Anesth Analg 102:896-899, 2006

4. Golshani K, Ferrell A, Zomorodi A, Smith TP, Britz GW: A review of the management of posterior communicating artery aneurysms in the modern era. Surg Neurol Int 1:88, 2010

5. Legatt AD: Case 39, in Intraoperative Neurophysiology: Interactive Case Studies. New York: Demos Medical Publishing, 2015, p 7

6. Legatt AD: Intraoperative neurophysiologic monitoring: Some technical considerations. Am J EEG Technol 35:167200, 1995

7. Nuwer MR, Packwood JW: Somatosensory evoked potential monitoring with scalp and cervical recording, in Nuwer MR (ed): Intraoperative Monitoring of Neural Function. Handbook of Clinical Neurophysiology. Amsterdam: Elsevier, 2008, Vol 8, pp 180-189

8. Pollard ME, Little PW: Changes in carotid artery blood flow during anterior cervical spine surgery. Spine (Phila Pa 1976) 27:152-155, 2002

9. Rhoton AL Jr, Saeki N, Perlmutter D, Zeal A: Microsurgical anatomy of common aneurysm sites. Clin Neurosurg 26:248-306, 1979

10. Rothwell J, Burke D, Hicks R, Stephen J, Woodforth I, Crawford M: Transcranial electrical stimulation of the motor cortex in man: further evidence for the site of activation. $\mathbf{J}$ Physiol 481:243-250, 1994

11. Sloan TB, Ronai AK, Koht A: Reversible loss of somatosensory evoked potentials during anterior cervical spinal fusion. Anesth Analg 65:96-99, 1986

12. Yeh YC, Sun WZ, Lin CP, Hui CK, Huang IR, Lee TS: Prolonged retraction on the normal common carotid artery induced lethal stroke after cervical spine surgery. Spine (Phila Pa 1976) 29:E431-E434, 2004

\section{Disclosures}

The authors report no conflict of interest concerning the materials or methods used in this case or the findings specified in this paper.

\section{Author Contributions}

Conception and design: Legatt. Acquisition of data: Legatt, Laarakker, Nakhla, Altschul. Analysis and interpretation of data: all authors. Drafting the article: Legatt, Laarakker. Critically revising the article: all authors. Reviewed submitted version of manuscript: all authors.

\section{Supplemental Information}

\section{Previous Presentations}

This case report was presented in poster form at the 2016 Annual Meeting of the American Clinical Neurophysiology Society held in Orlando, Florida, on February 12-14, 2016.

\section{Correspondence}

Alan D. Legatt, Department of Neurology, Montefiore Medical Center, 111 East 210 St., Bronx, NY 10467. email: alegatt@ montefiore.org. 